\title{
Common and uncommon bilateral adult renal masses
}

\author{
Anjali Roy ${ }^{a}$, Ott Le ${ }^{b}$, Paul M. Silverman ${ }^{\text {b }}$, Vikas Kundra ${ }^{\text {b }}$ \\ ${ }^{a}$ Medical Diagnostic Imaging Group, 10835 N 25th Ave, Suite 240, Phoenix, AZ 85029-4751, USA; \\ ${ }^{b}$ University of Texas MD Anderson Cancer Center, Unit 1473, PO Box 301439, Houston, \\ TX 77230-1439, USA \\ Corresponding address: Ott Le, MD, University of Texas MD Anderson Cancer Center, Unit 1473, \\ PO Box 301439, Houston, TX 77230-1439, USA. \\ Email: ott.le@mdanderson.org
}

Date accepted for publication 5 March 2012

\begin{abstract}
Masses can involve the kidney unilaterally or bilaterally. The purpose of this article is to review common and uncommon adult renal masses that present bilaterally. Clinical and imaging findings are described. Renal masses that present in a bilateral fashion can have particular clinical and imaging characteristics and knowledge of their presentation enables appropriate diagnosis and management, especially in a multidisciplinary care setting. More commonly found bilateral renal masses that are discussed include metastasis, lymphoproliferative disorders, adult polycystic kidney disease, angiomyolipomas, renal infracts and renal abscesses. Less common bilateral renal masses include transitional cell carcinoma, oncocytoma, and hematomas.
\end{abstract}

Keywords: Bilateral adult renal masses; renal metastasis; renal lymphoma; adult polycystic kidney disease; renal angiomyolipomas; renal infracts; renal abscesses; transitional cell carcinoma; oncocytoma; renal hematomas.

\section{Introduction}

There are common and uncommon renal masses other than renal cell carcinoma that often present bilaterally. Indeed, $10-30 \%$ of renal masses are benign at surgery ${ }^{[1]}$. Resection and biopsy at certain times can be avoided when there are clinical and imaging characteristics that suggest the diagnosis.

The purpose of this article is to review the clinical aspects and imaging characteristics of common and uncommon adult bilateral renal masses, excluding renal cell carcinoma. Some such masses have characteristic clinical and imaging findings and knowledge of their presentation and imaging features can aide appropriate diagnosis and management, especially in a multidisciplinary care setting. Examples of bilateral renal masses to be discussed include: metastasis, lymphoma, leukemia, adult polycystic kidney disease, angiomyolipoma, renal infarction and renal abscess.

\section{Commonly found bilateral renal masses}

\section{Renal metastases}

Renal metastases are the most common malignant neoplasm of the kidney found at autopsy ${ }^{[2]}$. Renal metastases are commonly bilateral rather than unilateral and occur most commonly in the setting of late stage malignancy. They are usually asymptomatic. Hematuria or microhematuria is seen in only $12-31 \%$ of patients $^{[3]}$. Lung cancer is the most common malignancy to metastasize to the kidneys followed by breast cancer, gastric cancer and melanoma ${ }^{[3]}$. However, almost any late stage malignancy can metastasize to the kidneys.

Imaging findings of metastases are nonspecific. On computed tomography (CT), they often manifest as mildly enhancing, bilateral, small hypoattenuating masses $^{[4]}$ (Fig. 1). However, the appearance of metastases can vary and they may be unilateral and hypervascular,

This paper is available online at http://www.cancerimaging.org. In the event of a change in the URL address, please use the DOI provided to locate the paper. 


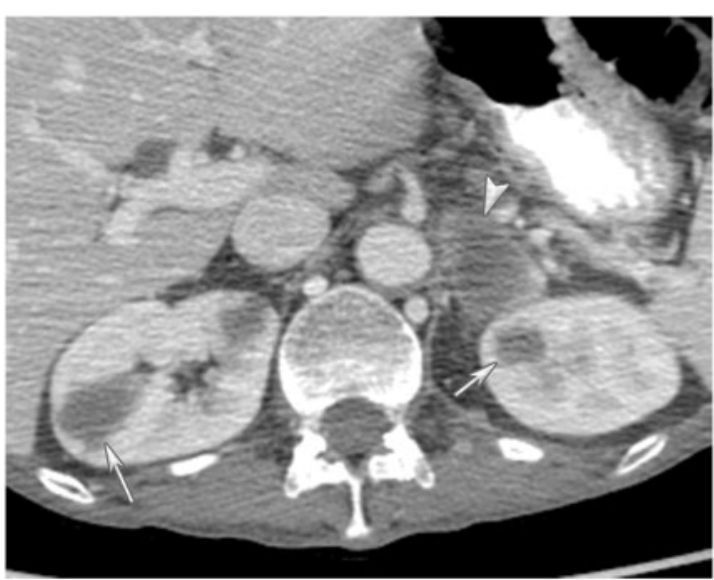

Figure 1 A 52-year-old man with non-small cell lung cancer. Metastatic disease to both kidneys manifests as poorly enhancing masses on CT imaging with intravenous contrast (arrows). Note also the additional metastatic lesion to the left adrenal gland (arrowhead).

with or without cystic components or calcifications. When imaging characteristics of metastases mimic those of renal cell carcinoma, the presence of known disseminated malignancy favors renal metastases. Tissue diagnosis is rarely needed in this setting. Treatment is directed at the primary cancer.

\section{Lymphoproliferative disorders}

\section{Lymphoma}

Renal lymphoma most often manifests as multiple bilateral renal masses and occasionally, nephromegaly. Unilateral renal lymphoma is less common, occurring $28 \%$ of the time in one study by Heiken et al. ${ }^{[5]}$. The genitourinary system is the second most common organ system affected by extranodal spread of lymphoma, after the hematopoietic and reticuloendothelial systems; the kidneys are the most commonly involved organs ${ }^{[6]}$. Extranodal involvement of the kidneys is found in $30 \%$ of patients with non-Hodgkin lymphoma ${ }^{[7]}$. Renal lymphoma usually occurs in the setting of widespread intermediate and high-grade B-cell type non-Hodgkin lymphoma or American Burkitt lymphoma ${ }^{[8]}$. Renal involvement by Hodgkin disease is rare and is seen in less than $1 \%$ of patients at presentation ${ }^{[9,10]}$.

Although renal lymphoma is common in patients with lymphomatous disease already seen elsewhere, primary renal lymphoma is rare. It seen in less than $1 \%$ of cases of extranodal lymphoma ${ }^{[11]}$. In the majority of cases, renal lymphoma is clinically asymptomatic and radiological detection seldom affects staging and treatment. Patients may present with flank pain, hematuria, fever and night sweats. Renal failure occurs with more advanced disease. Immunocompromised patients are at a significantly higher risk for developing lymphoma; and extranodal lymphoma, including renal lymphoma, is particularly common in this patient population.

Imaging findings of renal lymphoma include: multiple, poorly enhancing or hypoechoic renal masses, retroperitoneal masses directly invading the kidneys in a contiguous fashion, diffuse renal infiltration with bilateral renal enlargement and masses, and perirenal soft tissue masses, and increased metabolic activity on positron emission tomography (PET) (Fig. 2). Cystic lesions and tumors affecting the renal sinus and collecting system are uncommon. Unless the renal lesions manifest in the setting of widespread lymphoma, percutaneous biopsy is indicated to differentiate lymphoma from other lesions such as metastases, hypovascular renal cell carcinoma, urothelial carcinoma, or atypical infection. Chemotherapy, not surgery, is the treatment of choice for renal lymphoma.

\section{Leukemia}

Leukemia involving the kidneys is primarily seen in children. Patients who are at higher risk of extramedullary disease, such as those with T-cell acute lymphoblastic leukemia and M4 and M5 subtypes of acute myelogenous leukemia are more likely to have renal involvement $^{[12]}$. Up to $52 \%$ of patients with late stage leukemia can have renal involvement. However, they are less common than lymphoma when presenting as bilateral renal masses.

Patients are usually asymptomatic. Imaging findings include bilateral, low attenuation renal parenchymal abnormalities such as multiple small round masses, wedge-shaped lesions, geographic masses, and ill-defined areas of low attenuation ${ }^{[12]}$. PET imaging also demonstrates increased metabolic activity (Fig. 3). Leukemia may also appear as bilateral or unilateral nephromegaly $^{[12]}$. Renal leukemia may be indistinguishable from renal lymphoma and nephroblastomatosis on imaging. However, renal lymphoma has a greater propensity to involve the perinephric spaces. Again, treatment primarily involves chemotherapy, not surgery.

\section{Plasma cell neoplasia}

Plasma cell neoplasia is a malignant disorder involving differentiated B lymphocytes (plasma cells) that most often arise in bone either as a solitary lesion (plasmacytoma) or multiple lesions (multiple myeloma). Multiple myeloma and plasmacytoma of bone account for approximately $95 \%$ of plasma cell neoplasms. Multiple myeloma may involve extraskeletal sites, including the kidney, usually as microscopic foci of plasma cells. This has been reported in up to $17 \%$ of patients dying of multiple myeloma. Primary extramedullary plasmacytomas represent about $5 \%$ of plasma cell neoplasms and these most often arise in the upper respiratory tract. Rarely, plasmacytomas may arise in the renal interstitium ${ }^{[13]}$. In cases of isolated renal plasmacytoma, a monoclonal 
(a)

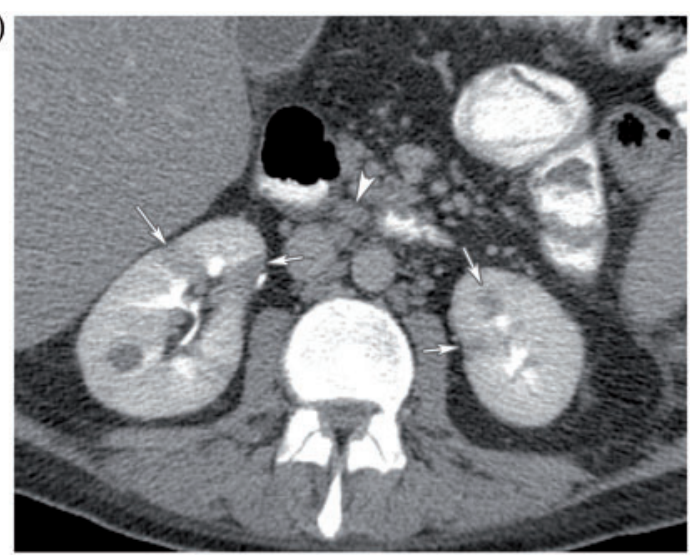

(b)

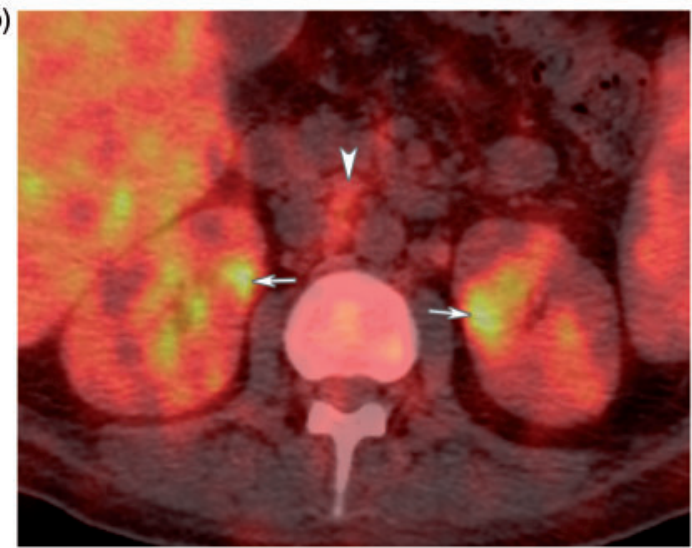

Figure 2 (a) A 30-year-old man with follicular lymphoma. CT with contrast reveals bilateral, multiple poorly enhancing renal masses from lymphomatous involvement (arrows). Note the associated retroperitoneal and mesenteric lymphadenopathy (arrowhead). (b) A 30-yearold man with follicular lymphoma. The bilateral cortical renal masses are metabolically active as seen with $\left[{ }^{18}\right.$ F $]$ fluorodeoxyglucose (FDG)-PET/CT imaging (arrows). Some of the associated retroperitoneal lymphadenopathy is slightly metabolically active (arrowhead).

immunoglobulin may be detected at serum electrophoresis or there may be Bence-Jones proteinuria, findings that establish the diagnosis. If the tumor does not manufacture immunoglobulin, the diagnosis may not be able to be established preoperatively.

Renal plasmacytomas may appear well circumscribed or infiltrative, and may be difficult to distinguish from other renal primary tumors by imaging alone, but history is helpful. Treatment also involves primarily chemotherapy, not surgery.

Again, renal leukemia and renal plasmacytoma occur less often than renal lymphoma as bilateral renal masses.

\section{Adult polycystic kidney disease}

Autosomal dominant polycystic disease (ADPKD) is a multisystemic disease characterized by progressive (a)

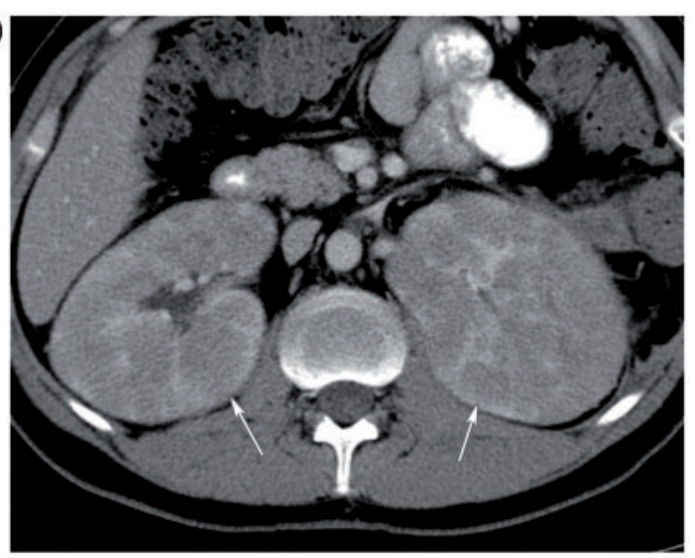

(b)

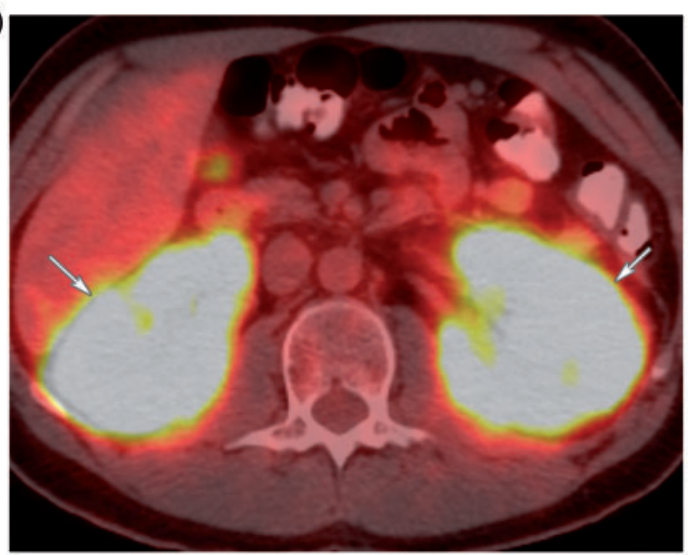

Figure 3 (a) A 25-year-old man with acute lymphoblastic leukemia, with renal involvement. Both kidneys are enlarged with multiple, poorly enhancing masses as seen on CT with intravenous contrast (arrows). (b) A 25-yearold man with acute lymphoblastic leukemia, with renal involvement. The renal masses are diffusely metabolically active as seen on $\left[{ }^{18}\right.$ F]FDG-PET/CT imaging (arrows).

formation and enlargement of renal cysts as well as cysts in other organs such as the liver, pancreas and kidney. It is responsible for $6-10 \%$ of end-stage renal disease in adults in the United States and Europe. It has an incidence of 1 to 2:1000 live births with 17\% of cases initially presenting in one kidney, but most cases progress to both kidneys ${ }^{[14]}$. The genes for ADPKD are primarily PKD1, located on chromosome 6, responsible for ADPKD type 1, and PKD2 located on chromosome 4, responsible for ADPKD type 2. Although the two subtypes have the same clinical and imaging characteristics, ADPKD type 2 is associated with a younger age of onset of end-stage renal disease. ADPKD is usually detected in the third or fourth decade of life. However, renal cysts can be detected during childhood or in utero $^{[15]}$. The renal cysts are epithelial cysts that originate as saccular outpouchings from renal tubules that eventually separate from the tubules and progressively enlarge in size, leading to massive renal enlargement. The cysts also invoke interstitial changes leading to 
interstitial fibrosis. Renal involvement can present clinically as flank pain, hypertension, hematuria or renal failure $^{[16]}$.

Cystic renal involvement can be detected by ultrasonography or other cross-sectional imaging. Imaging findings include bilateral, enlarged kidneys with multiple cysts of varying size some of which are hemorrhagic and appear hyperdense on precontrast $\mathrm{CT}$ and hyperintense on T1weighted MR images (Fig. 4). The cysts may have peripheral, curvilinear calcifications and distort and splay the pelvicalyceal system. However, they do not enhance with contrast. Extrarenal manifestations include hepatic, pancreatic and splenic cysts, intracranial and coronary aneurysms, mitral valve prolapse, and possibly spontaneous arterial dissections ${ }^{[17]}$.

Treatment options include symptomatic management, dialysis and renal transplantation. End-stage renal disease is the leading cause of morbidity and mortality in ADPKD. Other causes of mortality include cardiovascular pathology, infections and subarachnoid hemorrhage from ruptured cerebral berry aneurysms.

\section{Angiomyolipoma}

Angiomyolipomas are the most common benign renal mesenchymal neoplasms. They have an incidence of $0.3-3 \%$ of renal neoplasm ${ }^{[18]}$. Not only are they commonly found as unilateral renal masses, but also as bilateral renal masses. bilateral renal masses are especially seen in patients with tuberous sclerosis; small, multicentric, asymptomatic angiomyolipomas occur in $80 \%$ of patients ${ }^{[19]}$.

Angiomyolipomas demonstrate characteristic fat attenuation on CT. On magnetic resonance imaging (MRI), they are hyperintense on T1-weighted images, hypointense on T2-weighted images, and show loss of signal on fat-suppressed images (Fig. 5).

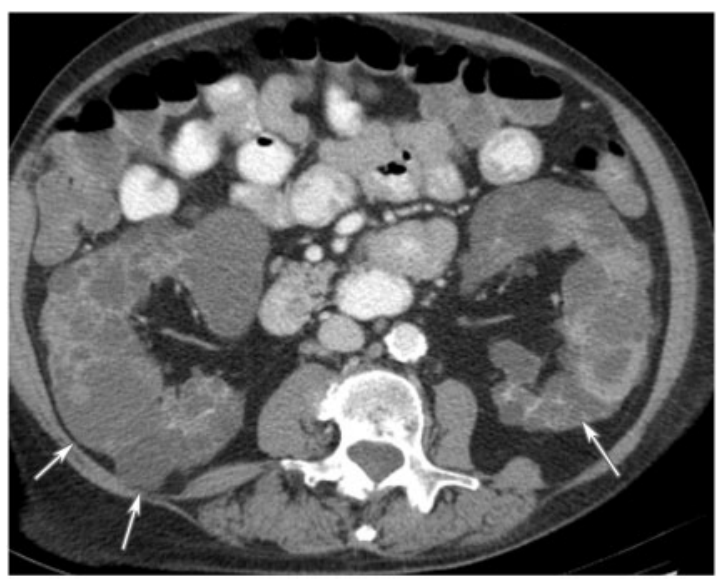

Figure 4 A 66-year-old woman with autosomal dominant polycystic kidney disease (ADPKD). CT with intravenous contrast reveals enlargement of both kidneys with innumerable cysts from ADPKD (arrows).
Hemorrhage represents a known complication, occurring in up to $13 \%$ of cases ${ }^{[20]}$. Management of asymptomatic angiomyolipomas less than $4 \mathrm{~cm}$ entails annual or semi-annual follow-up; symptomatic lesions are treated with surgical resection or selective embolization.

\section{Renal infarcts}

Renal infarcts may be caused by ischemia as a result of emboli, trauma, or thrombosis such as secondary to vasculitis. The incidence of renal infarction has been documented at $1.4 \%$ in a study of 13,411 autopsies $^{[21]}$. Renal infarcts can be asymptomatic or may present with acute flank pain, hematuria, proteinuria or leukocytosis. Renal infarction may be global or segmental $^{[22]}$. Global renal infarction results from compromised renal arterial supply or venous drainage from a hypercoagulable state, trauma or aortic dissection. In comparison, segmental infarcts are usually embolic or related to vasculitis. If embolic, endocarditis is a common source of emboli.

Global renal infarction manifests as decreased enhancement of the renal parenchyma, often with a simultaneous rim of cortical enhancement from the capsular arteries, also called the cortical rim sign. Segmental infarcts are bilateral or unilateral, peripherally located, wedge-shaped areas of low attenuation and decreased enhancement. They are also best depicted in the pyelographic phase of enhancement ${ }^{[23]}$ (Fig. 6).

\section{Renal abscess}

Renal abscess can affect a single kidney or both simultaneously. An incidence of 1-10 cases per 10,000 hospital admissions has been documented. It occurs in the setting of acute pyelonephritis secondary to obstruction with ascending organism such as Escherichia coli or Proteus

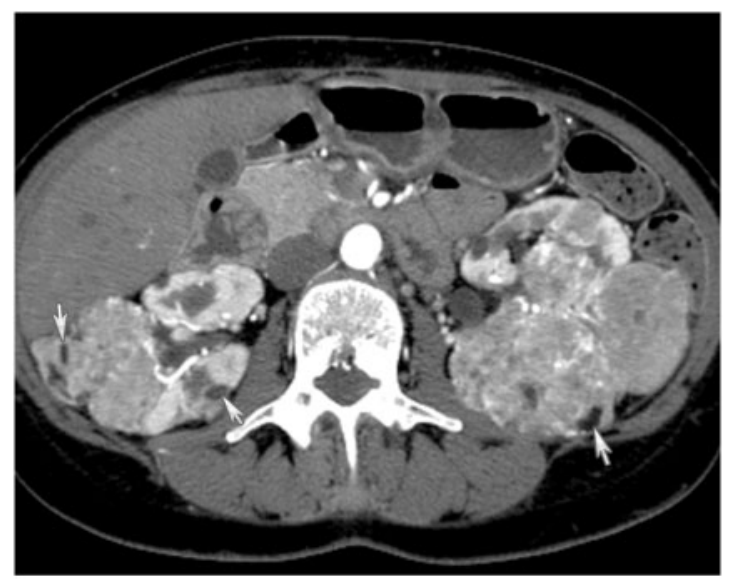

Figure 5 A 51-year-old woman with tuberous sclerosis. CT with intravenous contrast reveals bilateral enhancing renal masses containing foci of fat attenuation consistent with angiomyolipomas (arrows). 


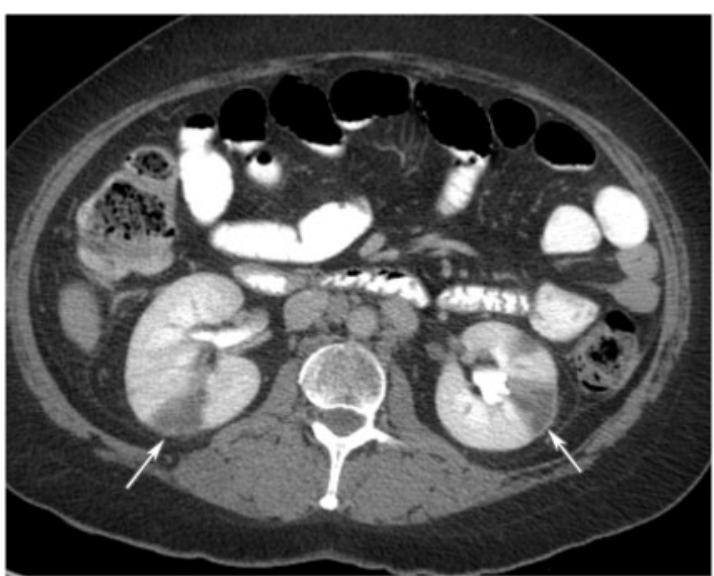

Figure 6 A 35-year-old woman with cervical cancer. New wedge-shaped low attenuation in both kidneys with slight cortical rim enhancement suggestive of bilateral renal infarcts as seen on $\mathrm{CT}$ with intravenous contrast (arrows).

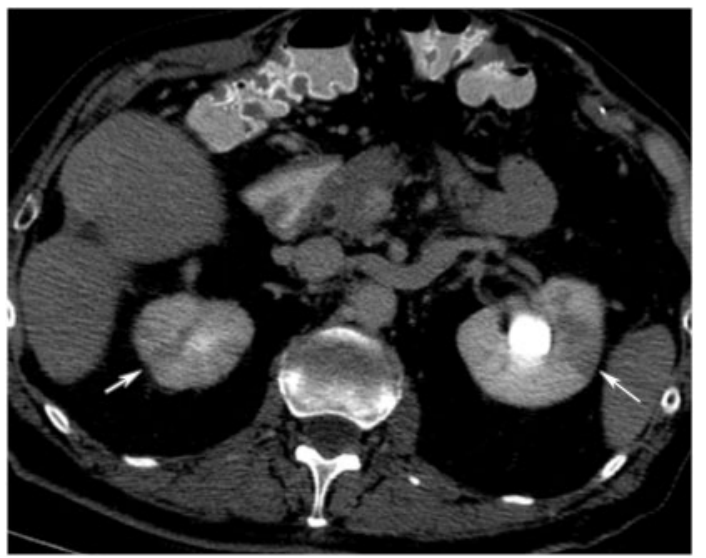

Figure 7 A 72-year-old man with metastatic rectal cancer. Bilateral low attenuations in both kidneys in this patient are due to pyelonephritis as seen on CT with intravenous contrast (arrows).

(Fig. 7). Hematogenous spread or direct extension from an extrarenal infectious process can also result in renal abscess formation.

Renal abscess is recognized on imaging as a focal fluid collection with enhancing walls on CT or MRI with contrast. Pockets of gas may also be present. On ultrasonography, they are hyperechoic in the acute phase and isoechoic to hypoechoic in the subsequent phase. Internal septations, debris or pockets of gas can also be seen. Left untreated, the abscess can extend into and cause thickening of the Gerota fascia and spread to the psoas muscle. Antibiotics and drainage are the common means of treatment.

\section{Less commonly found bilateral renal masses}

\section{Transitional cell carcinoma}

Although transitional cell carcinoma (TCC) is most commonly encountered in the urinary bladder, up to $10 \%$ of transitional cell carcinomas occur in the upper urinary tract $^{[24]}$. They can involve one kidney or both kidneys. Only $2-4 \%$ of renal TCCs are found to be bilateral, however. They can be multicentric and synchronous or metachronous. Multicentric TCCs are common and portend poor prognosis. Patients with TCC typically present with microscopic or gross hematuria. Upper tract TCC typically occurs in the sixth or seventh decade of life, with a male preponderance of $3: 1$. Known risk factors include smoking, chemical carcinogens such as aniline, benzidine and azo dyes, cyclophosphamide, heavy caffeine consumption, and analgesic abuse, especially phenacetin $^{[24]}$.

Renal TCC most frequently arises in the extrarenal part of the pelvis. TCCs involving the kidney usually manifests as single or multiple, smooth, irregular or stippled filling defects, or as strictures of the renal pelvis that may extend into the proximal ureter (Fig. 8). On ultrasonography, renal TCCs typically appear as soft tissue masses of variable echogenicity in the echogenic renal sinus fat with or without generalized or focal hydronephrosis. They are also known to invade the renal parenchyma in an infiltrative pattern.

Radical treatment such as nephroureterectomy for bilateral TCCs is not recommended. Instead, a conservative approach to resection such as ureteroscopic and percutaneous techniques or renal sparing surgery are advocated $^{[25]}$.

\section{Oncocytoma}

Oncocytoma has been described as the most common benign renal tumor accounting for $3-10 \%$ of all renal tumors. Oncocytoma is usually unilateral and solitary. However, they rarely can occur in a bilateral fashion. Familial oncocytomas are rare, but do occur in patients with Hogg-Dube syndrome ${ }^{[26]}$. The syndrome is again rare, the incidence of which is unknown. It is a manifestation of a genetic disorder with predisposition to cancerous and noncancerous tumors of the kidneys, cysts of the kidneys and lung, and noncancerous tumors of the hair follicles. Bilateral oncocytomas have been described in this syndrome.

On imaging, they are classically described as low attenuation masses with spoke-wheel enhancement and a central scar, but rarely present as such. They cannot be clearly distinguished from renal cell carcinoma on imaging or commonly on biopsy, which may necessitate resection or watchful waiting (Fig. 9). 
(a)

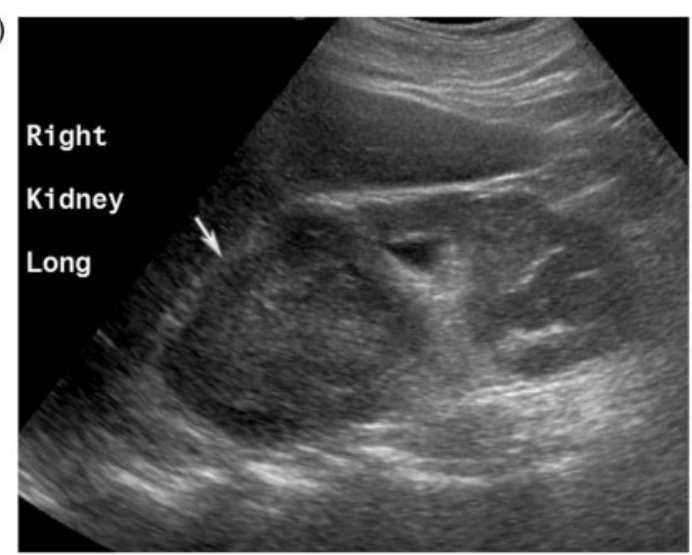

(b)

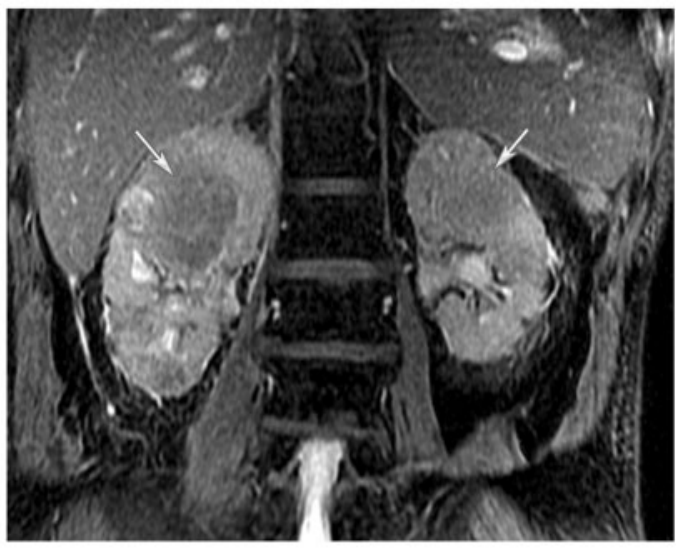

(c)

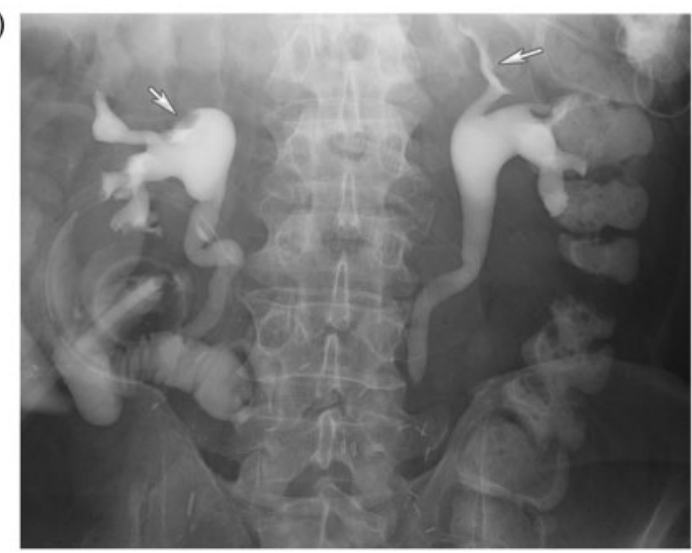

Figure 8 (a) A 64-year-old man with TCC. Ultrasonography reveals heterogeneous, echogenic masses invading and distorting the right kidney (arrow). (b) A 64-year-old man with transitional cell carcinoma. MRI coronal Fiesta image shows the bilateral renal mass from TCC invading the renal parenchyma (arrows). (c) A 64-year-old man with transitional cell carcinoma. A loopogram shows irregular filling defects in the upper pole calices of both kidneys from TCC (arrows).

\section{Hematoma}

Renal hematomas are more likely to occur unilaterally, but on rare occasions can be bilateral. Renal hematomas can be subscapsular, perinephric and infrequently have

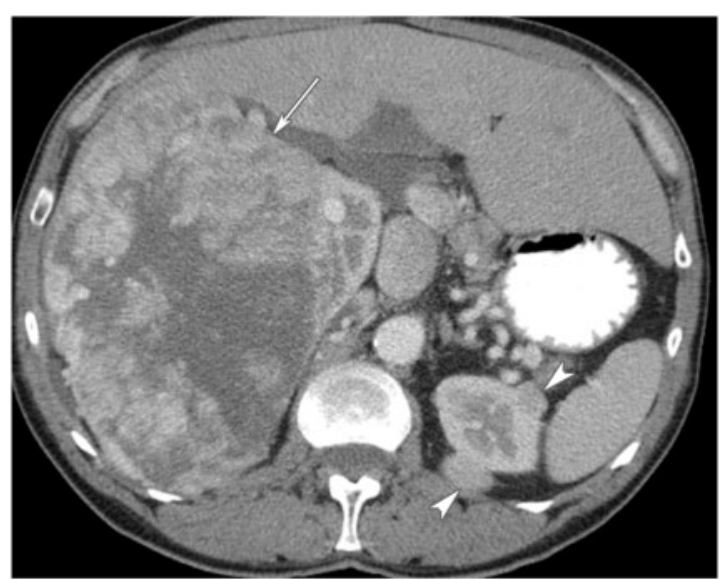

Figure 9 A 61-year-old man with bilateral oncocytomas. CT of the abdomen with intravenous contrast demonstrates a large, heterogeneous, enhancing mass growing from the right kidney, pathologically proven to be oncocytoma (arrow). Smaller solid homogenously enhancing masses in the left kidney are also oncocytomas (arrowhead).

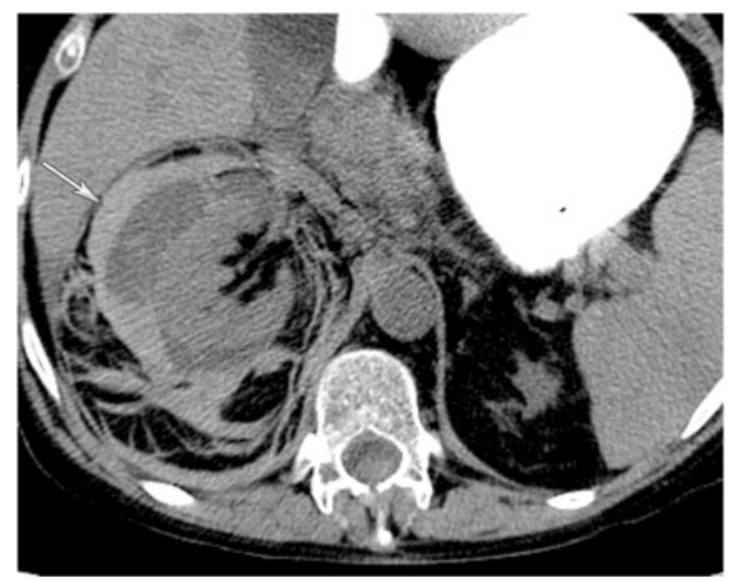

Figure 10 A 46-year-old woman with hypereosinophilic syndrome. An acute right perinephric hematoma is seen as a hyperattenuating fluid collection on CT without intravenous contrast (arrow).

a mass-like appearance. Underlying neoplasm, vasculitides, infection, or bleeding dyscrasia should be sought for spontaneous renal hematomas. On CT without intravenous contrast, hematomas are hyperdense in the acute phase then become isodense to hypodense over time (Fig. 10).

\section{Conclusion}

A variety of benign and malignant masses can involve the kidneys in a bilateral fashion. Some are more common 


\section{Table 1 Bilateral renal masses}

\begin{tabular}{|c|c|}
\hline $\begin{array}{l}\text { Bilateral renal } \\
\text { masses }\end{array}$ & Distinguishing features \\
\hline \multicolumn{2}{|c|}{ More common bilateral renal masses } \\
\hline Renal metastases & $\begin{array}{l}\text { Occur in setting of known primary cancer in } \\
\text { late stage disease }\end{array}$ \\
\hline $\begin{array}{l}\text { Lymphoproliferative } \\
\text { disorders }\end{array}$ & $\begin{array}{l}\text { Low attenuation renal masses } \\
\text { Renal enlargement } \\
\text { Renal lymphomas have other sites of involve- } \\
\text { ment, such as perinephric masses }\end{array}$ \\
\hline $\begin{array}{l}\text { Adult polycystic } \\
\text { kidney disease }\end{array}$ & $\begin{array}{l}\text { Enlarged kidneys with multiple cysts } \\
\text { Pancreatic and hepatic cysts } \\
\text { Hemorrhagic cysts } \\
\text { Multisystem disease }\end{array}$ \\
\hline Angiomyolipoma & $\begin{array}{l}\text { Contains fat } \\
\text { Commonly found in tuberous sclerosis }\end{array}$ \\
\hline Renal infarcts & $\begin{array}{l}\text { Wedge shaped } \\
\text { Decreased enhancement } \\
\text { Cortical rim sign }\end{array}$ \\
\hline Renal abscess & $\begin{array}{l}\text { Fluid collection with peripheral enhancement } \\
\text { Perinephric inflammation and other signs of } \\
\text { pyelonephritis }\end{array}$ \\
\hline \multicolumn{2}{|c|}{ Less common bilateral renal masses } \\
\hline $\begin{array}{l}\text { Transitional cell } \\
\text { carcinoma }\end{array}$ & $\begin{array}{l}\text { Infiltrative pattern with invasion of renal sinus } \\
\text { fat and parenchyma } \\
\text { Filling defect, mural thickening or obstructed } \\
\text { calices }\end{array}$ \\
\hline Oncocytoma & $\begin{array}{l}\text { Benign slow growing mass } \\
\text { Classically, central scar with spoke-wheel } \\
\text { enhancement }\end{array}$ \\
\hline Hematoma & $\begin{array}{l}\text { No enhancement } \\
\text { Hyperdense when acute on noncontrast } \\
\text { enhanced CT } \\
\text { Look for underlying cause in spontaneous renal } \\
\text { hematomas } \\
\text { Becomes smaller with time }\end{array}$ \\
\hline
\end{tabular}

than others. For instance, metastatic disease and lymphoproliferative disorders are more commonly found in a bilateral fashion compared with TCCs or oncocytomas. Some of the bilateral renal masses can have clinical and imaging overlaps. However, knowledge of their characteristic features and clinical setting can aid in appropriate diagnosis and management.

\section{References}

[1] Kutikov A, Fossett LK, Ramchandani P, et al. Incidence of benign pathologic findings at partial nephrectomy for solitary renal mass presumed to be renal cell carcinoma on preoperative imaging. Urology 2006; 68: 737-740. doi:10.1016/j.urology. 2006.04.011.

[2] Hietala SO, Wahlqvist L. Metastatic tumors to the kidney. A postmortem, radiologic and clinical investigation. Acta Radiol Diagn (Stockh) 1982; 23: 585-591.

[3] Wagle DG, Moore RH, Murphy GP. Secondary carcinomas of the kidney. J Urol 1975; 114: 30-32.

[4] Choyke PL, White EM, Zeman RK, Jaffe MH, Clark LR. Renal metastases: clinicopathologic and radiologic correlation. Radiology 1987; 162: 359-363.

[5] Heiken JP, Gold RP, Schnur MJ, King DL, Bashist B, Glazer HS. Computed tomography of renal lymphoma with ultrasound correlation. J Comput Assist Tomogr 1983; 7: 245-250. doi:10.1097/00004728-198304000-00007.

[6] Sheth S, Ali S, Fishman E. Imaging of renal lymphoma: patterns of disease with pathologic correlation. Radiographics 2006; 26: 1151-1168. doi:10.1148/rg.264055125.

[7] Kose F, Sakalli H, Mertsoylu H, et al. Primary renal lymphoma: report of four cases. Onkologie 2009; 32: 200-202. doi:10.1159/ 000203331.

[8] Strauss S, Libson E, Schwartz E, et al. Renal sonography in American Burkitt lymphoma. AJR Am J Roentgenol 1986; 146: $549-552$.

[9] Chepuri NB, Strouse PJ, Yanik GA. CT of renal lymphoma in children. AJR Am J Roentgenol 2003; 180: 429-431.

[10] Guermazi A, Brice P, de Kerviler EE, et al. Extranodal Hodgkin disease: spectrum of disease. Radiographics 2001; 21: 161-179.

[11] Stallone G, Infante B, Manno C, Campobasso N, Pannarale G, Schena FP. Primary renal lymphoma does exist: case report and review of the literature. J Nephrol 2000; 13: 367-372.

[12] Hilmes MA, Dillman JR, Mody RJ, Strouse PJ. Pediatric renal leukemia: spectrum of CT imaging findings. Pediatr Radiol 2008; 38: 424-430. doi:10.1007/s00247-007-0741-5.

[13] Jaspan T, Gregson R. Extra-medullary plasmacytoma of the kidney. Br J Radiol 1984; 57: 95-97. doi:10.1259/0007-128557-673-95.

[14] Mea Bisceglia. Renal cystic diseases: a review. Adv Anat Pathol 2006; 13: 26-56.

[15] Ravine D, Gibson RN, Walker RG, Sheffield LJ, KincaidSmith P, Danks DM. Evaluation of ultrasonographic diagnostic criteria for autosomal dominant polycystic kidney disease 1 . Lancet 1994; 343: 824-827. doi:10.1016/S0140-6736(94) 92026-5.

[16] Braun WE. Autosomal dominant polycystic kidney disease: emerging concepts of pathogenesis and new treatments. Cleveland Clin J Med 2009; 76: 97-104. doi:10.3949/ccjm.76a.gr001.

[17] Demetriou K, Tziakouri C, Anninou K, et al. Autosomal dominant polycystic kidney disease-type 2. Ultrasound, genetic and clinical correlations. Nephrol Dial Transplant 2000; 15: 205-211. doi:10.1093/ndt/15.2.205.

[18] Steiner MS, Goldman SM, Fishman EK, Marshall FF. The natural history of renal angiomyolipoma. J Urol 1993; 150: 1782-1786.

[19] Casper KA, Donnelly LF, Chen B, Bissler JJ. Tuberous sclerosis complex: renal imaging findings. Radiology 2002; 225: 451-456. doi:10.1148/radiol.2252011584.

[20] Koh KB, George J. Radiological parameters of bleeding renal angiomyolipoma. Scand J Urol Nephrol 1996; 30: 265-268. doi:10.3109/00365599609182303.

[21] Hoxie H, Coggin CB. Renal infarction: statistical study of two hundred and five cases and detailed report of an unusual case. Arch Intern Med 1940; 65: 587. doi:10.1001/archinte.1940. 00190090124007.

[22] Lee EY. CT imaging of mass-like renal lesions in children. Pediatr Radiol 2007; 37: 896-907. doi:10.1007/s00247-007-0548-4.

[23] Kawashima A, Sandler CM, Ernst RD, Tamm EP, Goldman SM, Fishman EK. CT evaluation of renovascular disease 1 . Radiographics 2000; 20: 1321-1340.

[24] Hall MC, Womack S, Sagalowsky AI, Carmody T, Erickstad MD, Roehrborn CG. Prognostic factors, recurrence, and survival in transitional cell carcinoma of the upper urinary tract: a 30-year experience in 252 patients. Urology 1998; 52: 594-601. doi:10.1016/S0090-4295(98)00295-7.

[25] Oosterlinck W, Solsona E, van der Meijden APM, et al. EAU guidelines on diagnosis and treatment of upper urinary tract transitional cell carcinoma. Eur Urol 2004; 46: 147-154. doi:10.1016/j.eururo.2004.04.011.

[26] Choyke PL, Glenn GM, Walther MM, Zbar B, Linehan WM. Hereditary renal cancers. Radiology 2003; 226: 33-46. doi:10.1148/radiol.2261011296. 